\title{
TITLE:
}

\section{Hydrogel-mediated semiconductor wafer bonding}

$\operatorname{AUTHOR}(S):$

Kishibe, Kodai; Tanabe, Katsuaki

CITATION:

Kishibe, Kodai ...[et al]. Hydrogel-mediated semiconductor wafer bonding. Applied Physics Letters 2019, 115(8): 081601.

ISSUE DATE:

2019-08-19

URL:

http://hdl.handle.net/2433/243889

\section{RIGHT:}

This article may be downloaded for personal use only. Any other use requires prior permission of the author and AIP Publishing. This article appeared in Kishibe K. and Tanabe K., Applied Physics Letters 115:8 and may be found at

https://doi.org/10.1063/1.5096540.; The full-text file will be made open to the public on 21 August 2020 in accordance with publisher's 'Terms and Conditions for Self-Archiving' 


\title{
Hydrogel-mediated semiconductor wafer bonding
}

Cite as: Appl. Phys. Lett. 115, 081601 (2019); doi: 10.1063/1.5096540

Submitted: 18 March 2019 - Accepted: 2 August 2019 .

Published Online: 21 August 2019

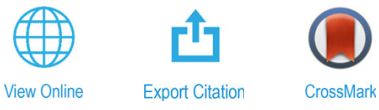

Kodai Kishibe and Katsuaki Tanabe

AFFILIATIONS

Department of Chemical Engineering, Kyoto University, Nishikyo, Kyoto 615-8510, Japan

${ }^{a)}$ Author to whom correspondence should be addressed: tanabe@cheme.kyoto-u.ac.jp

\begin{abstract}
The concept of hydrogel-mediated semiconductor wafer bonding was proposed and demonstrated in this work. The unique property of hydrogels was utilized to simultaneously realize high mechanical stability, electrical conductivity, and optical transparency in semiconductor interfaces. The high applicability of this method for rough surfaces to be bonded was also demonstrated, owing to the soft, deformable interfacial contact agent to be solidified in the bonding process. Furthermore, the bonding experiments were carried out in ambient air at room temperature, which, therefore, provides cost and throughput advantages in device production. In addition, the developed bonding technique was used for demonstrating the fabrication and operation of solar cell devices, with current paths across the bonded interfaces, which verified the method's practical applicability. Our semiconductor bonding and interfacial engineering scheme are expected to open up a pathway for simple, handy, and low-cost, but flexible and high-performance optoelectronic material integration.
\end{abstract}

Published under license by AIP Publishing. https://doi.org/10.1063/1.5096540

Semiconductor wafer bonding is a skillful fabrication method used in various electronics and photonics applications, ${ }^{1-5}$ in particular, to form high-crystalline-quality homo- and heterostructures of semiconductor materials with low defect densities, which are otherwise difficult to obtain via the conventional growth methods ${ }^{6-8}$ because of the existence of insulating oxide interlayers or crystalline lattice mismatches. Therefore, semiconductor wafer bonding is seen as a promising technique for the fabrication of high-performance semiconductor optoelectronics and has been employed to generate a variety of devices, such as light-emitting diodes, ${ }^{9-11}$ lasers, ${ }^{12-16}$ photodetectors, ${ }^{17,18}$ and solar cells. ${ }^{19-22}$

For many optoelectronic device applications, interfacial electrical conductivity and optical transparency are required or preferable, which can be fulfilled by the direct semiconductor-to-semiconductor bonding scheme. ${ }^{19-22}$ Nevertheless, the wafer surface condition for the conventional direct bonding and bonding mediated by solid-state materials such as oxides ${ }^{3,13}$ or metals ${ }^{9,14}$ is severely restricted because the surface roughness and particulates worsen the interfacial stability and conductivity. Semiconductor wafer bonding mediated by polymer materials can mitigate such a surface-condition restriction but hardly provides electrical conductivity and optical transparency simultaneously. For example, poly(3,4-ethylenedioxythiophene): poly(styrenesulfonate) (known as PEDOT:PSS) and polyaniline, representatives of conductive polymers, provide good electrical conductivity, but poor optical transparency. ${ }^{23}$ Transparent polymers such as polyimide, benzocyclobutene (known as BCB), and divinyl siloxane-bis-benzocyclobutene (DVS-BCB) have been applied for semiconductor wafer bonding, ${ }^{24-26}$ but are all electrically insulating.

Hydrogels have matrix structures generated by crosslinks of hydrophilic polymers and contain a substantial amount of water (60\%-90\%). Their high moisture content, self-healing ability, and three dimensional network structures are ideal for application in contact lenses, paper diapers, and supporting media for electrophoresis. Recently, hydrogel's flexibility and electrical conductivity have been utilized for the realization of regenerative medicine through the intensive fabrication of biological scaffolds and drag delivery systems. ${ }^{27-30}$ Furthermore, their bonding ability is a recent find. ${ }^{31-33}$ This study exploits the unique properties of hydrogels, including electrical conductance, optical transparency, adhesiveness, and flexibility, for semiconductor bonding. The bonding characteristics are compared for three types of representative hydrogels, namely, polyacrylamide (PAM), polyvinyl alcohol (PVA), and agarose (AGR). The hydrogel bonding scheme presented herein especially benefits from high surface-roughness, particulate tolerances, optical transmittance, and electrical conductivity.

We used single-side-polished p-type $\mathrm{Si}$ wafers (thickness: $280 \mu \mathrm{m}$; crystalline plane orientation: (100); dopant: boron; doping level: $10^{19} \mathrm{~cm}^{-3}$ ); their polished surfaces were coated with a photoresist film for protection as they were diced into $8 \mathrm{~mm} \times 8 \mathrm{~mm}$ squares. The diced wafers were submerged in acetone for $5 \mathrm{~min}$ for removal of the photoresist film, followed by their submersion in a $9 \%$ 
hydrofluoric acid solution for $1 \mathrm{~min}$ so as to remove the native oxide layer. In terms of coating, it would be better to coat hydrogel materials onto hydrophilic surfaces, rather than hydrophobic ones such as those undergone hydrofluoric-acid treatments. However, we carried out the oxide-removing hydrofluoric treatment in this study for the purpose of minimizing the interfacial electrical resistivity. In addition, the hydrogel materials to be coated with that we prepared in this study are relatively viscous and therefore can be smoothly coated even onto hydrophobic semiconductor surfaces. Three types of hydrogels were prepared: (a) 2.5-w/v\% PAM aqueous solution (aq.) prepared through a mixture of PAM powder with de-ionized water that was stirred well to prevent the aggregation of the adhesive PAM particles; (b) $12.5-w / v \%$ PVA aq.; and (c) $2.5-w / v \%$ AGR aq. The last two hydrogels need to be heated, and so they can be dissolved in water and, therefore, were prepared by mixing PVA and AGR powders with de-ionized water and stirring on a hot plate at $80^{\circ} \mathrm{C}$ and $130^{\circ} \mathrm{C}$, respectively. The prepared hydrogel was uniformly spin-coated on the Si wafer. To improve the reproducibility of the experimental results, the spin-coating process was repeated three times. The hydrogel-coated Si piece was bonded to a bare Si piece under a uniaxial pressure of $0.1 \mathrm{MPaG}$ in ambient air at room temperature. Note that $2.5-w / v \%$ AGR aq. was solidified immediately after it was dropped on the wafer, and therefore, the AGR-dropped Si piece was immediately bonded to a bare Si piece without spin coating.

Detaching normal stresses were measured for the bonded samples to represent the bonded interfacial mechanical strength. For electrical measurements, metal electrodes comprising an $\mathrm{Au}-\mathrm{Ge}-\mathrm{Ni}$ alloy (80:10:10 wt. \%) and pure Au with thicknesses of 30 and $150 \mathrm{~nm}$ were sequentially deposited to both the outer sides of the bonded Si pieces, respectively. Afterward, the current-voltage characteristics across the bonded interfaces were measured, as depicted in Fig. S1. Furthermore, for optical transmission measurements, hydrogel-coated glasses instead of the Si wafers were prepared in the same way as above. Si wafers were used in this study as the representative of the semiconductor material, but the concept is easily extensible to other semiconductors, as a number of wafer bonding experimental demonstrations between dissimilar materials have been reported so far. ${ }^{13,19,20,34}$

Figure 1 shows a typical cross-sectional scanning electron microscopy image of the bonded Si/PAM/Si interface. As seen in this image, the wafers were in uniform and firm contact with each other at a mechanical stability sufficient to endure the cleavage of the bonded pair sample. In addition, infrared transmission images of the bonded interfaces (Fig. S2) confirmed the global contact with no interfacial void for the entire $8 \mathrm{~mm} \times 8 \mathrm{~mm}$-square sample area and with a PAM-thickness uniformity around $200 \mathrm{~nm} / \mathrm{mm}$. Thus, we have

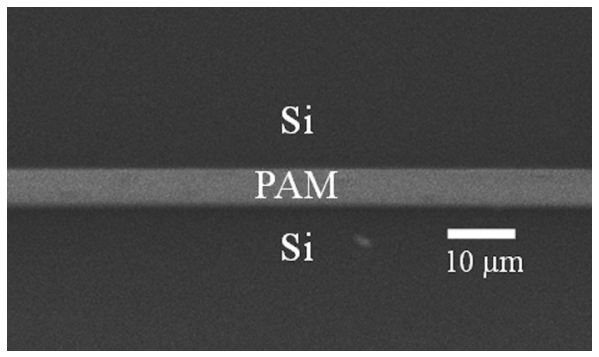

FIG. 1. Cross-sectional scanning electron microscopy image of the PAM-mediatedbonded interface. introduced a uniform hydrogel film between Si wafers for realizing hydrogel-mediated semiconductor bonding. The hydrogen bonds from PAM may cause the adhesion to semiconductor surfaces. ${ }^{35,36}$ More specifically, hydrogen bonds are presumably formed between the $-\mathrm{NH}_{2}$ groups of PAM and the Si surface terminated by $-\mathrm{OH}$ groups due to the water contained in PAM. ${ }^{35}$ The PAM film had an approximate thickness of $5 \mu \mathrm{m}$, larger than typical diameters of particles in a regular, noncleanroom environment $(<3 \mu \mathrm{m})$. Therefore, even in the presence of such particles on wafers, hydrogel films change their own morphology to enclose the particles and result in particulate tolerances. Moreover, the epitaxially grown semiconductor top surface has generally much smaller roughness than the thickness of such hydrogel films, indicating that our hydrogel-mediated bonding scheme would likewise mitigate the smoothness requirements on the surface roughness, for example, of epitaxial semiconductor materials to be bonded to fabricate optoelectronic devices.

We carried out peel strength tests to evaluate the bonding strength of each Si/hydrogel/Si interface. Mechanical strengths of the bonded interfaces mediated by PAM, PVA, and AGR were measured to be 180,290 , and $120 \mathrm{kPa}$, respectively. All samples were thus sufficiently strong to endure a series of optoelectronic device manufacturing processes and user operation, especially the sample with $12.5-\mathrm{w} / \mathrm{v} \%$ PVA aq., which had the highest mechanical strength of the bonded interface of nearly $300 \mathrm{kPa}$. This result suggests that as the concentration of hydrogel increases, the mechanical strength increases with the formation of a highly dense interlayer.

In addition, in view of the relatively weak interfacial bonding strengths measured for the hydrogel-mediated bonding scheme in this study relative to the bonding strengths over $1 \mathrm{MPa}$ for wellestablished, existing state-of-the-art semiconductor wafer bonding methods, the potential use of the hydrogel bonding as a temporary adhesive comes to mind. Namely, the adhesion and possible detachment by hydrogels can be utilized for the protection of semiconductor wafer surfaces during storage or carriage $e^{37}$ and for the transfer and printing of semiconductor thin films onto arbitrary substrates. ${ }^{4,14}$

Figure 2 shows the current-voltage characteristics across the bonded interfaces. As seen from the straight current-voltage curves, ohmic electrical conductance was obtained for all the hydrogels tested as the bonding agent. Note that the data in Fig. 2 include all series resistance through the sample; therefore, we independently determined the contact resistance of the metal electrode/semiconductor

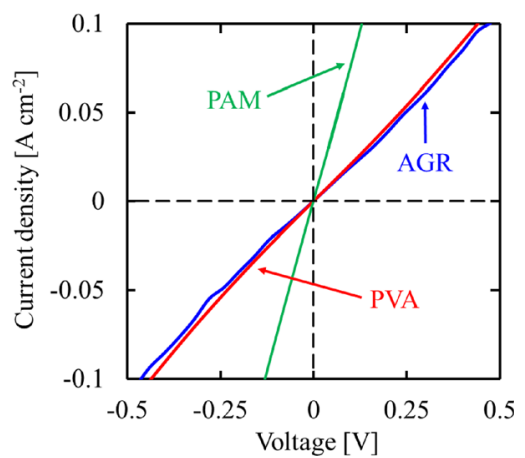

FIG. 2. Current-voltage characteristics of the bonded interfaces mediated by PAM, $P V A$, and $A G R$. 
interfaces by the transmission line method and then determined the nominal resistivity at the bonded interface by subtracting it from the slope of the current-voltage curve. The determined interfacial electrical resistances of PVA- and AGR-bonded interfaces were 4.6 and $4.9 \mathrm{ohm} \mathrm{cm}^{2}$, respectively, whereas the $\mathrm{Si} / \mathrm{PAM} / \mathrm{Si}$ interface had the lowest electrical resistance of $0.84 \mathrm{ohm} \mathrm{cm}^{2}$. PAM, a hydrophilic polymer material, can contain water inside, and the water-originated ions act as electrical carriers to induce the electrical conductance. ${ }^{36}$ Such an electrical conductivity value obtained can be considered preferable for many optoelectronic device applications such as solar cells. ${ }^{38,39}$ To achieve lower electrical resistance, we can optimize the preparation process conditions, such as the hydrogel species, concentration, and interlayer thickness, depending on the demand of each application. Particularly, the interlayer hydrogel film thickness, which is an important bonding factor, can be controlled by the choice of various parameters, including the wettability of the semiconductor surface, the spin-coating rotation velocity, and the coating repetition. Moreover, blending of conductive polymers, such as polyacetylene and polythiophene, ${ }^{40,41}$ or carbon nanotubes ${ }^{28}$ with the hydrogels may be effective for further enhancement of electrical conductivity.

Figure 3 shows the optical transmittance spectra of the hydrogel films. Each of the presented optical transmittance spectra is generated by normalizing the raw transmittance spectral data of the sample with the hydrogel film by those of the glass plate alone without the hydrogel film. Spectral discontinuity was incidentally observed around $960 \mathrm{~nm}$, due to the switching of the spectrometers. All kinds of hydrogels tested in this study were observed to exhibit high transmissivities, higher than $95 \%$, for the entire wavelength region measured, through the violet to the near-infrared region. Particularly, PAM exhibited remarkable optical transmissivities higher than $99 \%$ for the entire spectral region. In this way, semiconductor interfaces produced by our hydrogelmediated bonding approach would have negligibly small optical losses, which are good indicators for various optoelectronic applications, such as multijunction solar cell devices. Overall, PAM was found to provide the highest electrical conductance and optical transmittance, with a good mechanical stability and, therefore, was the material employed for further bonding experiments described in the subsequent sections.

Let us discuss the potential optical reflection at the bonded interfaces because of the refractive-index mismatch between hydrogel and semiconductor materials. Hydrogel materials are known to have refractive indices of about 1.5 for optical frequencies, as also demonstrated by the low reflectivity on glass in Fig. 3, while typical inorganic

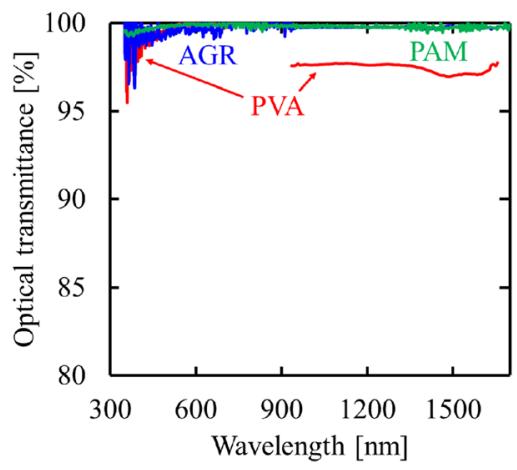

FIG. 3. Optical transmittance spectra of the PAM, PVA, and AGR films. semiconductor materials such as $\mathrm{Si}, \mathrm{GaAs}$, and $\mathrm{InP}$ have refractive indices about 3.5. As a rough estimate based on these representative refractive-index values, a single hydrogel/semiconductor interface would have a reflectivity of $16 \%$. Therefore, a hydrogel-bonded semiconductor interface with a hydrogel interlayer with a thickness sufficiently larger than the optical wavelength would have a net reflectivity of $29 \%$. Such a degree of interfacial optical reflectance may not be preferable for a number of optoelectronic applications. However, for the interlayer thicknesses around the order of the optical wavelengths, the reflectivity varies in an oscillating manner on the interlayer thickness and the optical wavelength and, therefore, can be controlled by the hydrogel thickness, depending on each application. In addition, such an interfacial reflectance could be even positively utilized, for example, for current matching in multijunction solar cells, by the reflectivity tuning by the hydrogel interlayer thickness. Moreover, the reflectance can be eliminated by employing a hydrogel thickness sufficiently smaller than the optical wavelength, along with an account for the trade-off with the surface-roughness tolerance.

For many device fabrication procedures, postprocessing after the bonding is mandatory. In the postprocesses, thermal treatments, such as contact annealing, are often required. Therefore, we additionally investigated the thermal impact on the bonding, to examine the limitation of the hydrogel-bonding approach. Figures S3 and S4 show the current-voltage characteristics across the bonded interfaces and the optical transmittance spectra of the hydrogel films, respectively, after annealing at various temperatures, and Table S1 shows the determined interfacial electrical resistivity values. The annealing processes were carried with a hot plate in ambient air at various temperatures for $3 \mathrm{~h}$. In these series of experiments, a single bonded sample was used, for each of the electrical and optical measurements, repeatedly being tested sequentially from the lower- to higher-temperature conditions, to clearly observe the evolution in the characteristics by circumventing the characteristic fluctuation among the samples. As observed in Figs. S3 and S4 and Table S1, little degradation in the electrical conductivity and optical transparency occurs below $150^{\circ} \mathrm{C}$, while significant degradation occurs above $200^{\circ} \mathrm{C}$. Therefore, in practical optoelectronic applications of the hydrogel-mediated bonding, it may become important to employ thermal postprocessing with temperatures below $150^{\circ} \mathrm{C}$ to sustain desirable interfacial characteristics.

To demonstrate the versatility of our hydrogel-mediated bonding scheme, we carried out bonding experiments for rough semiconductor ( $\mathrm{Si}$, this case) surfaces. We intentionally roughened the $\mathrm{Si}$ wafer surface by dipping it in a chemical etching solution comprising hydrofluoric acid, nitric acid, and de-ionized water ( $\mathrm{HF}: \mathrm{HNO}_{3}: \mathrm{H}_{2} \mathrm{O}$ $=23: 30: 47 \mathrm{wt}$. \%) for $5 \mathrm{~s}$ at room temperature. The obtained root mean square roughness of the etched, originally polished $\mathrm{Si}$ surface was $1.2 \mu \mathrm{m}$. We tried the direct semiconductor bonding ${ }^{19,42}$ with no interfacial mediating agent for the roughened Si surfaces at $300{ }^{\circ} \mathrm{C}$ and $600^{\circ} \mathrm{C}$ for $3 \mathrm{~h}$, for which we bonded well for nonroughened surfaces, but we were not able to form any bond at all. This failure result was necessary because, for example, the conventional direct wafer bonding method ${ }^{19-22,42,43}$ is known for being unsuccessful for bonding surface roughness larger than $10 \mathrm{~nm}$ (see Fig. S1 and Table S1 in Ref. 42). By contrast, we bonded between the roughened surfaces by the PAM-mediated bonding scheme, with an interfacial bonding strength of $320 \mathrm{kPa}$. In this way, we have experimentally demonstrated that the hydrogel mitigates roughness tolerance for surfaces 
to be bonded owing to the soft, deformable bonding agent, verifying one of the advantages of our approach. This effect of the hydrogelmediated bonding is important in application use, whereas in many cases, bonding surfaces generally exhibit a certain degree of roughness as those surfaces are typically top surfaces after some fabrication procedures, such as chemical treatments, crystal growth, or micro/ nano processes. Incidentally, the obtained interfacial bonding strength in this test, $320 \mathrm{kPa}$, was interestingly even higher than that for the case of the nonroughened, smooth surfaces, $180 \mathrm{kPa}$, for the PAM-mediated bonding. Such bonding stability enhancement by roughened bonding surfaces can be attributed to an increase in the area of contact between $\mathrm{Si}$ and the hydrogel due to the surface roughness. It is worth mentioning that we conducted the experiments entirely in a noncleanroom, regular experimental room, thus demonstrating the high particulate tolerance of our hydrogel bonding scheme, in contrast to direct bonding.

Using the hydrogel-mediated bonding technique, we fabricated Si solar cells bonded to Si wafers to demonstrate the applicability of our bonded semiconductor interface in optoelectronic devices. Si solar cells were prepared by thermal diffusion of phosphorus $\left(10^{19}-10^{20} \mathrm{~cm}^{-3}\right)$ into one side of the surface region of a doubleside-polished epi-ready $p$-type $\mathrm{Si}\langle 100\rangle$ wafer doped with boron (doping concentration of $\sim 1 \times 10^{16} \mathrm{~cm}^{-3}$ ). After the phosphorus thermal diffusion, the boron doping concentration on the other surface was increased to the level of $10^{19}-10^{20} \mathrm{~cm}^{-3}$ by ion implantation, to provide a sufficient electrical conductance at the bonded interface. The $p$-type side of the Si solar cell wafer was bonded to a bare $\mathrm{Si}$ wafer (the same wafer used for the above bonding investigations) mediated by PAM under the same process conditions as those in the bonding investigation. A front grid contact on top of the Si cell and a bottom contact on the back of the bare Si wafer were formed with the $\mathrm{Au} / \mathrm{Au}-\mathrm{Ge}-\mathrm{Ni}$ metal material in the same manner as in the bonding investigation. Note that in this electrode configuration, current was allowed to pass through the bonded interface during the solar cell operation. Therefore, these solar cell fabrication and operation tests are suitable for evaluating the validity of our bonding scheme for optoelectronic device applications. For comparison, we prepared a pristine reference solar cell sample from the same Si solar cell wafer, with the same top and bottom electrodes but standing alone, not bonded with a bare Si wafer. We used this performance comparison to estimate the loss in power conversion efficiency by the electrical resistance at the bonded interface.

Figure 4 shows the light current-voltage characteristics of the best bonded and best reference cells under AM1.5 G, 1-sun $\left(100 \mathrm{~mW} \mathrm{~cm}^{-2}\right)$ illumination. The bonded cell performed a little lower than the reference cell (e.g., the energy-conversion efficiencies $\eta$ of the bonded and reference cells were $7.23 \%$ and $8.00 \%$, respectively). However, our fabricated cells exhibited a certain degree of statistical fluctuations in their performances. The inset of Fig. 4 presents the average solar cell performance parameters (open-circuit voltage $V_{O C}$, short-circuit current density $J_{S C}$, fill factor $F F$, and $\eta$ ) statistically averaged for each of the bonded and reference cells. As observed in this statistics, the performances of the bonded cells were highly comparable to those of the unbonded reference cells. For example, the average $\eta$ values of the bonded and reference cells were $6.81 \%$ and $6.73 \%$, respectively; the bonded cells were slightly better than the reference cells. Therefore, the bonding process and the bonded interface did not degrade the solar cell and its performance, validating

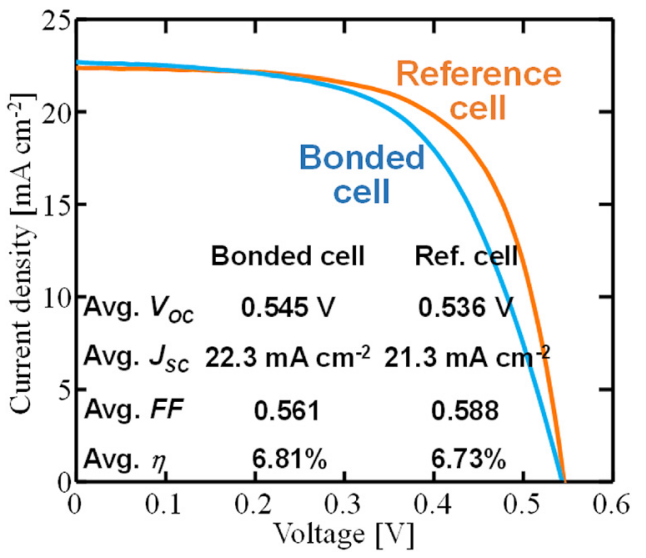

FIG. 4. Light current-voltage characteristics of the highest-efficiency bonded and reference unbonded $\mathrm{Si}$ solar cells, and (inset) average $V_{O C}, J_{S C}, F F$, and $\eta$ values, under the AM1.5 G, 1-sun illumination.

the suitability of our bonding scheme for optoelectronic device applications, regarding both handiness and performance.

In this study, we proposed and experimentally demonstrated a hydrogel-mediated semiconductor wafer bonding, as a concept to provide a high-performance yet handy bond formation method. Owing to the distinctive characteristics of the hydrogel materials, high mechanical stability, electrical conductivity, optical transparency, surfaceroughness, and particulate tolerances were simultaneously acquired in the bonded semiconductor interfaces. We carried out the bonding experiments in ambient air at room temperature, in which heating was not required, unlike in conventional growth methods and direct semiconductor wafer bonding processes. Therefore, we do not encounter the risk of superfluously damaging the semiconductor material or retarding the production cycle, while providing cost advantages during manufacturing. For simplicity, we incidentally used Si wafers as the representative semiconductor material, but our concept is extendable to other semiconductors, as numerous wafer bonding experimental demonstrations between dissimilar semiconductor materials are reported thus far. ${ }^{13,19,34,42}$ Furthermore, we demonstrated the fabrication and operation of solar cells using the developed bonding technique, with current paths across the bonded interfaces, verifying the validity of our scheme in practical device applications. Our hydrogelmediated bonding and interfacial engineering scheme may be useful for flexible and low-cost production of high-performance integrated electronic and photonic devices.

\section{See the supplementary material for Figs. S1-S4 and Table S1.}

This study was supported, in part, by the Japan Society for the Promotion of Science (JSPS) and the Nanotechnology Platform Project sponsored by the Ministry of Education, Culture, Sports, Science and Technology (MEXT), Japan.

\section{REFERENCES}

1J. B. Lasky, "Wafer bonding for silicon-on-insulator technologies," Appl. Phys. Lett 48, 78-80 (1986).

${ }^{2}$ S. Noda, K. Tomoda, N. Yamamoto, and A. Chutinan, "Full three-dimensional photonic bandgap crystals at near-infrared wavelengths," Science 289, 604-606 (2000). 
${ }^{3}$ G. K. Celler and S. Cristoloveanu, "Frontiers of silicon-on-insulator," J. Appl. Phys 93, 4955-4978 (2003).

${ }^{4}$ M. Madsen, K. Takei, R. Kapadia, H. Fang, H. Ko, T. Takahashi, A. C. Ford, M. H. Lee, and A. Javey, "Nanoscale semiconductor "X" on substrate "Y"Processes, devices, and applications," Adv. Mater 23, 3115-3127 (2011).

${ }^{5} \mathrm{~T}$. Naito and K. Tanabe, "Fabrication of Si/graphene/Si double heterostructures by semiconductor wafer bonding towards future applications in optoelectronics," Nanomater 8, 1048 (2018).

${ }^{6} \mathrm{H}$. Kroemer, T. Y. Liu, and P. M. Petroff, "GaAs on Si and related systems: Problems and prospects," J. Cryst. Growth 95, 96-102 (1989).

${ }^{7}$ M. Sugo, Y. Takanashi, M. M. Al-Jassim, and M. Yamaguchi, "Heteroepitaxial growth and characterization of InP on Si substrates," J. Appl. Phys. 68, 540-547 (1990).

${ }^{8}$ J. Z. Li, J. Bai, J. S. Park, B. Adekore, K. Fox, M. Carroll, A. Lochtefeld, and Z. Shellenbarger, "Defect reduction of GaAs epitaxy on $\mathrm{Si}$ (001) using selective aspect ratio trapping," Appl. Phys. Lett 91, 021114 (2007).

${ }^{9}$ W. S. Wong, T. Sands, N. W. Cheung, M. Kneissl, D. P. Bour, P. Mei, L. T. Romano, and N. M. Johnson, " $\mathrm{In}_{\mathrm{x}} \mathrm{Ga}_{1-\mathrm{x}} \mathrm{N}$ light emitting diodes on $\mathrm{Si}$ substrates fabricated by Pd-In metal bonding and laser lift-off," Appl. Phys. Lett 77, 2822-2824 (2000)

${ }^{10}$ J. Chun, K. J. Lee, Y. C. Leem, W. M. Kang, T. Jeong, J. H. Baek, H. J. Lee, B. J. Kim, and S. J. Park, "Vertically stacked color tunable light-emitting diodes fabricated using wafer bonding and transfer printing," ACS Appl. Mater. Interfaces 6, 19482-19487 (2014).

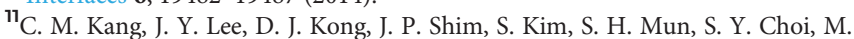
D. Park, J. Kim, and D. S. Lee, "Hybrid full-color inorganic light-emitting diodes integrated on a single wafer using selective area growth and adhesive bonding," ACS Photon 5, 4413-4422 (2018).

${ }^{12}$ J. Van Campenhout, P. Rojo-Romeo, P. Regreny, C. Seassal, D. Van Thourhout, S. Verstuyft, L. Di Cioccio, J. M. Fedeli, C. Lagahe, and R. Baets, "Electrically pumped InP-based microdisk lasers integrated with a nanophotonic silicon-oninsulator waveguide circuit," Opt. Express 15, 6744-6749 (2007).

${ }^{13}$ K. Tanabe, M. Nomura, D. Guimard, S. Iwamoto, and Y. Arakawa, "Room temperature continuous wave operation of InAs/GaAs quantum dot photonic crystal nanocavity laser on silicon substrate," Opt. Express 17, 7036-7042 (2009).

${ }^{14}$ S. Palit, J. Kirch, G. Tsvid, L. Mawst, T. Kuech, and N. M. Jokerst, "Low-threshold thin-film III-V lasers bonded to silicon with front and back side defined features," Opt. Lett 34, 2802-2804 (2009).

${ }^{15}$ D. Liang, X. Huang, G. Kurczveil, M. Fiorentino, and R. G. Beausoleil, "Integrated finely tunable microring laser on silicon," Nat. Photon 10, 719-722 (2016).

${ }^{16}$ G. Crosnier, D. Sanchez, S. Bouchoule, P. Monnier, G. Beaudoin, I. Sagnes, R. Raj, and F. Raineri, "Hybrid indium phosphide-on-silicon nanolaser diode," Nat. Photon 11, 297-300 (2017).

${ }^{17}$ H. Park, A. W. Fang, R. Jones, O. Cohen, O. Raday, M. N. Sysak, M. J. Paniccia, and J. E. Bowers, "A hybrid AlGaInAs-silicon evanescent waveguide photodetector," Opt. Express 15, 6044-6052 (2007).

${ }^{18} \mathrm{~L}$. Chen, P. Dong, and M. Lipson, "High performance germanium photodetectors integrated on submicron silicon waveguides by low temperature wafer bonding," Opt. Express 16, 11513-11518 (2008).

${ }^{19} \mathrm{~K}$. Tanabe, A. Fontcuberta i Morral, H. A. Atwater, D. J. Aiken, and M. W. Wanlass, "Direct-bonded GaAs/InGaAs tandem solar cell," Appl. Phys. Lett 89, 102106 (2006)

${ }^{20}$ F. Dimroth, M. Grave, P. Beutel, U. Fiedeler, C. Karcher, T. N. D. Tibbits, E. Oliva, G. Siefer, M. Schachtner, A. Wekkeli, A. W. Bett, R. Krause, M. Piccin, N. Blanc, C. Drazek, E. Guiot, B. Ghyselen, T. Salvetat, A. Tauzin, T. Signamarcheix, A. Dobrich, T. Hannappel, and K. Schwarzburg, "Wafer bonded four-junction GaInP/GaAs//GaInAsP/GaInAs concentrator solar cells with 44.7\% efficiency," Prog. Photovolt 22, 277-282 (2014).

${ }^{21}$ P. T. Chiu, D. C. Law, R. L. Woo, S. B. Singer, D. Bhusari, W. D. Hong, A. Zakaria, J. Boisvert, S. Mesropian, R. R. King, and N. H. Karam, "Direct semiconductor bonded 5J cell for space and terrestrial applications," IEEE J. Photovoltaics 4, 493-497 (2014).

${ }^{22}$ S. Essig, C. Allebé, T. Remo, J. F. Geisz, M. A. Steiner, K. Horowitz, L. Barraud, J. S. Ward, M. Schnabel, A. Descoeudres, D. L. Young, M. Woodhouse, M. Despeisse, C. Ballif, and A. Tamboli, "Raising the one-sun conversion efficiency of III-V/Si solar cells to $32.8 \%$ for two junctions and $35.9 \%$ for three junctions," Nat. Energy 2, 17144 (2017).

${ }^{23} \mathrm{~N}$. Matsuki, Y. Irokawa, Y. Nakano, and M. Sumiya, " $\pi$-conjugated polymer/ GaN schottky solar cells,” Sol. Energy Mater. Sol. Cells 95, 284-287 (2011).

${ }^{24}$ S. Matsuo, K. Tateno, T. Nakahara, H. Tsuda, and T. Kurokawa, "Use of polyimide bonding for hybrid integration of a vertical cavity surface emitting laser on a silicon substrate," Electron. Lett 33, 1148-1149 (1997).

${ }^{25}$ M. Lamponi, S. Keyvaninia, C. Jany, F. Poingt, F. Lelarge, G. de Valicourt, G. Roelkens, D. Van Thourhout, S. Messaoudene, M. J. Fedeli, and H. G. Duan, "Low-threshold heterogeneously integrated InP/SOI lasers with a double adiabatic taper coupler," IEEE Photonics Technol. Lett 24, 76-78 (2012).

${ }^{26}$ D. Inoue, J. Lee, K. Doi, T. Hiratani, Y. Atsuji, T. Amemiya, N. Nishiyama, and S. Arai, "Room-temperature continuous-wave operation of GalnAsP/lnP lateral-current-injection membrane laser bonded on Si substrate," Appl. Phys. Express 7, 072701 (2014).

${ }^{27}$ E. Montanari, S. Capece, C. D. Meo, M. Meringolo, T. Coviello, E. Agostinelli, and P. Matricardi, "Hyaluronic acid nanohydrogels as a useful tool for BSAO immobilization in the treatment of melanoma cancer cells," Macromol. Biosci 13, 1185-1194 (2013).

${ }^{28}$ S. Ahadian, J. Ramon-Azcon, M. Estili, X. Liang, S. Ostrovidov, H. Shiku, M. Ramalingam, K. Nakajima, Y. Sakka, and H. Bae, "Hybrid hydrogels containing vertically aligned carbon nanotubes with anisotropic electrical conductivity for muscle myofiber fabrication," Sci. Rep. 4, 4271 (2015).

${ }^{29} \mathrm{~J}$. Li and D. J. Mooney, "Designing hydrogels for controlled drug delivery," Nat. Rev. Mater. 1, 16071 (2016).

${ }^{30}$ H. Shigemitsu, T. Fujisaku, W. Tanaka, R. Kubota, S. Minami, K. Urayama, and I. Hamachi, "An adaptive supramolecular hydrogel comprising selfsorting double nanofibre networks," Nat. Nanotechnol 13, 165-172 (2018).

${ }^{31}$ S. Rose, A. Prevoteau, P. Elziere, D. Hourdet, A. Marcellan, and L. Leibler, "Nanoparticle solutions as adhesives for gels and biological tissues," Nature 505, 382-385 (2014).

${ }^{32}$ H. Yuk, T. Zhang, S. Lin, G. Alberto Parada, and X. Zhao, "Tough bonding of hydrogels to diverse non-porous surfaces," Nat. Mater. 15, 190-196 (2016).

${ }^{33}$ D. Wirthl, R. Pichler, M. Drack, G. Kettlguber, R. Moser, R. Gerstmayr, F. Hartmann, E. Bradt, R. Kaltseis, C. M. Siket, S. E. Schausberger, S. Hild, S. Bauer, and M. Kaltenbrunner, "Instant tough bonding of hydrogels for soft machines and electronics," Sci. Adv. 3, el700053 (2017).

${ }^{34} \mathrm{~K}$. Tanabe, K. Watanabe, and Y. Arakawa, "Flexible thin-film InAs/GaAs quantum dot solar cells," Appl. Phys. Lett. 100, 192102 (2012).

${ }^{35}$ H. G. Nam, M. G. Nam, P. J. Yoo, and J. Kim, "Hydrogen bonding-based strongly adhesive coacervate hydrogels synthesized using poly(N-vinylpyrrolidone) and tannic acid," Soft Matter 15, 785-791 (2019).

${ }^{36} \mathrm{Q}$. Zhang, X. Liu, L. Duan, and G. Gao, "Ultra-stretchable wearable strain sensors based on skin-inspired adhesive, tough and conductive hydrogels," Chem. Eng. J. 365, 10-19 (2019).

${ }^{37} \mathrm{Q}$. Tong, R. Gafiteanu, and U. Goesele, "Reversible silicon wafer bonding for surface protection: Wafer-enhanced debonding," J. Electrochem. Soc. 139, L101-L102 (1992).

${ }^{38}$ P. R. Sharps, M. L. Timmons, J. S. Hills, and J. L. Gray, "Wafer bonding for use in mechanically stacked multi-bandgap cells," Proceedings of the 26th IEEE Photovoltaics Specialists Conference, Anaheim (1997), pp. 895-898.

${ }^{39}$ S. Yoshidomi, J. Fukukawa, M. Hasumi, and T. Sameshima, "Mechanical stacking multi junction solar cells using transparent conductive adhesive," Energy Procedia 60, 116-122 (2014).

${ }^{40}$ S. I. Na, S. S. Kim, J. Jo, and D. Y. Kim, "Efficient and flexible ITO-free organic solar cells using highly conductive polymer anodes," Adv. Mater. 20, 4061-4067 (2008).

${ }^{41}$ D. A. Mengistie, M. A. Ibrahem, P. C. Wang, and C. W. Chu, "Highly conductive PEDOT:PSS treated with formic acid for ITO-free polymer solar cells," ACS Appl. Mater. Interfaces 6, 2292-2299 (2014).

${ }^{42} \mathrm{~K}$. Tanabe, K. Watanabe, and Y. Arakawa, "III-V/Si hybrid photonic devices by direct fusion bonding," Sci. Rep. 2, 349 (2012).

${ }^{43}$ V. Vincent Larrey, G. Mauguen, F. Fournel, D. Radisson, F. Rieutord, C. Morales, C. Bridoux, and H. Moriceau, "Adhesion energy and bonding wave velocity measurements,” ECS Trans. 75, 145-152 (2016). 